\title{
Physical, Neural, and Mental Timing
}

\author{
Wim van de Grind \\ Helmholtz Research School and Department of Functional Neurobiology, Utrecht University, \\ Utrecht, The Netherlands \\ E-mail: W.A.vandeGrind@bio.uu.nl
}

\begin{abstract}
The conclusions drawn by Benjamin Libet from his work with collegues on the timing of somatosensorial conscious experiences has met with a lot of praise and criticism. In this issue we find three examples of the latter. Here I attempt to place the divide between the two opponent camps in a broader perspective by analyzing the question of the relation between physical timing, neural timing, and experiential (mental) timing. The nervous system does a sophisticated job of recombining and recoding messages from the sensorial surfaces and if these processes are slighted in a theory, it might become necessary to postulate weird operations, including subjective back-referral. Neuroscientifically inspired theories are of necessity still based on guesses, extrapolations, and philosophically dubious manners of speech. They often assume some neural correlate of consciousness (NCC) as a part of the nervous system that transforms neural activity in reportable experiences. The majority of neuroscientists appear to assume that the NCC can compare and bind activity patterns only if they arrive simultaneously at the NCC. This leads to a search for synchrony or to theories in terms of the compensation of differences in neural delays (latencies). This is the main dimension of the Libet discussion. Examples from vision research, such as "temporal-binding-by-synchrony" and the "flash-lag" effect, are then used to illustrate these reasoning patterns in more detail. Alternatively one could assume symbolic representations of time and space (symbolic "tags") that are not coded in their own dimension (not time in time and space in space). Unless such tags are multiplexed with the quality message (tickle, color, or motion), one gets a binding problem for tags. One of the hidden aspects of the discussion between Libet and opponents appears to be the following. Is the NCC smarter than the rest of the nervous system, so that it can solve the problems of local sign (e.g., "where is the event"'?) and timing (e.g., "when did it occur?" and "how long did it last?'") on its own, or are these pieces of information coded symbolically early on in the system? A supersmart NCC appears to be the assumption of Libet's camp (which includes Descartes, but also mystics). The wish to distribute the smartness evenly across all stages of processing in the nervous system (smart recodings) appears to motivate the opponents. I argue that there are reasons to side with the latter group. ๑ 2002 Elsevier Science (USA)
\end{abstract}

\section{SOME CONCEPTUAL PRELIMINARIES}

As described and cited in detail in the accompanying papers by Gomes (2002), Pocket (2002), and Trevena and Miller (2002), Libet and collegues have done an extensive series of experiments on the mental timing of somatosensory experiences

The author thanks his collegues Jan Koenderink, Ans van Doorn, Martin Lankheet, and Richard van Wezel for inspiring discussions on the topics of this article and for critically reading earlier drafts.

Address correspondence and reprint requests to W. A. van de Grind, Department of Functional Neurobiology and Helmholtz School, Utrecht University, Padualaan 8, 3584 CH Utrecht, The Netherlands. Fax: +31302542219. 
and intentional decisions to move relative to the physical timing of worldly correlates. The explanatory hypotheses of Libet et al. rather than (or more than) the empirical findings are called into question by the critics. They are as follows: (1) conscious experiences of sensory events lag half a second behind their physical causes, but are subjectively referred back to the physical time of occurrence; and (2) the decision to move is taken unconsciously about $500 \mathrm{~ms}$ before the movement, but the conscious intention to move occurs only $200 \mathrm{~ms}$ before the movement. These theses are about the relation between physical timing and mental (experienced) timing. To place the discussion in perspective I look at the "intervening" role of nervous processes and thus broaden Libet's problem to the question of the relation between physical, neural, and mental timing. I take my examples mainly from vision research, but show how they relate to Libet's problem. Moreover, the visual "flash-lag" effect discussed below (Section 3.3) plays a role in Libet's experiments and forces us to shift the intentional decision to move some $80 \mathrm{~ms}$ back in time. Let us start with some general preliminaries.

In line with the 1600-year-old ideas of St. Augustine, J. J. Gibson entitled a talk at a meeting in 1973 (cited by Pöppel, 1978) as "Events Are Perceivable, but Time Is Not." Indeed, physical time is a concept in our theories about the world that is made tangible by detecting and counting physical events ( $1 \mathrm{~s}$ is $9,192,631,770$ cesium atom state changes). To measure time and time intervals in physics one needs the concepts of simultaneity and order of events. Relativity theory teaches us that two events that are simultaneous in one frame of reference are not simultaneous in a second frame of reference that is moving. These ideas from physics can be helpful in thinking about time experience or about the simultaneity and order of events in such different domains as the outside world, the nervous system, and conscious experience. We have a problem that is analogous to (not identical to) the problem physicists face because we need either a theory or solid experimental data (or both) that can relate our three "frames of reference" in which physical events, neural events, and experiential events take place.

Intuitively it seems reasonable to study temporal relations, as in physics, on the basis of the fundamental concepts of simultaneity and order. That is, if the two events $\mathrm{A}$ and $\mathrm{B}$ are not simultaneous then we should be able to say if A comes before or after B. Mathematically it is clear that one is then building a kind of topology of time, defining operations like equality (simultaneous) and greater than or smaller than (order). The scientific question about "mental time" is then "What mathematical structures (topologies or geometries) can best describe our experimental findings regarding simultaneity and order of experienced events?" A term like "time perception" is awkward in the literal sense referred to by Gibson because there is admittedly no stuff called "time" that is perceived. However, external events as perceived or caused by an agent have their place in the agent's "mental time" or "experienced time" and this makes it convenient to speak of perceived time. Such a term does not violate the habit of speech in physics, where there is also no stuff called time that can be measured.

In the frame of reference of conscious experiences one can indeed judge simultaneity versus successiveness and compare durations of "mental events." A full taxonomy of experiences relating to time is more complex and a laudable first attempt at 
constructing one can be found in Pöppel (1978). Here I use a coarser grain, since we need to cast our nets wider than experience alone. If we want to ground mental time in neural time in physical time, the nervous system cannot be skipped. It intervenes between external time and experiential time since it is responsible for "processing,' that is, the reordering and recombination of signals from the outside world. Thus it transforms external time relations and, leaving out this hidden transformation, might lead to the necessity to ascribe "miracles" to our conscious processes.

In making a distinction between experience and neural processes I do not opt for Cartesian dualism. It merely recognizes the generally accepted neuroscientific fact that only a subset of all neuronal activities is directly reflected in conscious experiences. Many neural operations are not in themselves experienced, but intercede between the external world and experience (perception) or between experience (will and decision) and action. It is convenient to refer to the particular part or aspect of the nervous system that supports conscious experiences as the "neural correlate of consciousness,' or NCC. This allows us to disregard for the time being the "hard", problem of consciousness, how it is that some neural processes are not experienced while others are. With "mental' time I refer to the experiential side of those neuronal processes of the NCC that appear to interpret the temporal order of some of the other neuronal processes. No nonphysical (nonmeasurable) entities are assumed or implied. The term " mental time" is also convenient because one can measure temporal experiences (simultaneity, successiveness, and order) in themselves without referring to the nervous system (for examples see Pöppel, 1978).

It is often assumed implicitly (and sometimes explicitly) that the term " $N C C$ " refers to certain neurons or certain brain regions that are conveniently clustered together (e.g., the frontal cortex), but this is not necessary. One could also envisage certain distinct patterns of activity as NCC or a distributed set of small neuron clusters, each embodying specific experiences as a kind of "micro-NCC.' In the context of the discussion between Libet and his opponents (Section 2) this is less important. What matters primarily is that the NCC or micro-NCC responsible for the studied experience, e.g., a tickle of the skin or an experienced color, requires a specific time (latency) to become active after some preceding (nonconscious) neural process has finished its preparatory activity. This assumption allows us to follow the discussion about the relation between external time, neural time, and experiential time. In honor of Libet's work we could call the problem of the relation between external time and mental time 'Libet's problem.' The main thesis of Libet's opponents is that one needs to look at the intervening neural processes (outside the NCC) in order to make sense of the data gathered by Libet and co-workers. As clarified above, I also think that this is the way to go if we want to solve Libet's problem.

Gomes (1998, 2002), Churchland (1981a, 1981b), Glynn (1990, 1991), Dennett and Kinsbourne (1992), Pocket (2002), and Trevena and Miller (2002) all contribute to the goal of developing an explicit theory to solve Libet's problem. In Section 2 I focus on the main postulates of Libet's opponents concerning the perceptual aspects of his problem. What is the common core of the discussion and can we formulate this in terms of a neural network model, a mechanism, to prepare for empirical testing? The perceptual aspects of the problem have recently been studied and discussed quite extensively in vision research. These findings and ideas have not yet played a 
role in the ongoing discussion between Libet and his critics. In Section 3 I therefore briefly review some of this work and discuss how it relates to Libet's problem. Since so much is known about the neural mechanisms of vision, the problem of specifying "hidden" neural processes becomes more tangible and has been partly solved.

The relation between neural timing and mental timing is far from trivial and few theories exist to help us. In Section 4, I analyze the problem of coding time information in the nervous system. There is a strong analogy with the problem of coding spatial information in neural activity patterns: the local sign problem. Proper solutions to the local sign problem chase the homunculus out of the brain. Similarly, proper solutions to the mental timing problem should solve the "binding problem," a temporal version of the homunculus problem. I can of course indicate only in which direction solutions to these age-old problems can possibly be found and to what kinds of experimental approaches they lead. Finally in Section 5 I look at the discussion between Libet and his opponents on the timing of conscious intentions (and decisions) to act. Whether we like it or not, this requires a brief look at the possible causal role of consciousness and voluntary (willed) activity. No miracles or nonphysical processes or events are needed, but (nonmaterial) concepts like "time" will continue to be part of our theories, as will other mathematical (nonmaterial) structures.

\section{NEURAL MECHANISMS POSTULATED TO EXPLAIN LIBET'S FINDINGS}

Libet has concluded from measurements done by his group over the years that it takes about half a second before external events can reach conscious awareness, but that the resulting experience is "back-referred" in time, so that it appears to occur in approximate synchrony with the stimulus. That a conscious experience takes place $500 \mathrm{~ms}$ after its external cause is no problem for any theory. Consciousness need not be in a hurry because virtually all time-critical processes are automatic and nonconscious. What is a problem, of course, is a conscious experience that time shifts itself back to the earlier time of occurrence of some external event (a Baron von Münchhausen-like operation). Libet's opponents point out that Libet's data can easily be explained without such a weird assumption and that Occam therefore prohibits the adoption of Libet's back-referral hypothesis.

In this discussion the term "latency" figures prominently. It seems to me that Libet only wants to use the word "latency" to refer to an interval (measured in cesium clock ticks) between some external stimulus event caused by the experimenter and the overt response of the subject as solicited by the experimenter. He does not want to speculate about underlying hidden neural processes or the function of the various neural waystations between the outside world and the NCC. Only simultaneously measured EEG events are allowed to play a role in his theory, but these are in themselves not interpreted in terms of underlying neuronal mechanisms. On the contrary, in his theory they are only tied to mental events, so that we do not leave the domain of experiences, even while using EEG signals. Such a positivistic stance of refusing to postulate hidden entities in a theory has its merits. However, it often necessitates "'miracles," events with a hidden (and thus undiscussed) "cause." The most recent discussion between Libet and his critics brings this out quite clearly. In 
his positivistic approach Libet needs time juggling to tie perceptual and EEG phenomena together. To eliminate the juggling, Gomes $(1998,2002)$ and other critics of Libet's conclusions introduce hypothetical neural processes in their theory, but Libet (2000) sees them as ad hoc assumptions.

In his Figs. 1, 5, and 6 Gomes (1998) splits Libet's experimental latency into a minimum train duration and a "real latency," where the latter is a completely internal neural latency caused by an intervening integration stage with threshold. Libet (2000, p. 3) replies that "There is no experimental evidence or justification for this specific added time.' 'He then stipulates that if such intervening latency is assumed, the same latency should be added to all experimental findings so that it makes no difference in the conclusions. This illustrates Libet's reservations regarding speculations on possible underlying (intervening) processes. These reservations notwithstanding he is willing to assume that all pathways from the outside world to conscious experience should basically have the same properties, including latencies. In fact, without this specific assumption, for which there is also no experimental evidence, Libet's startling conclusions break down. If the latency between stimulus and response is split in two (or even more) terms, and this is done independently for the different pathways used in Libet's experiments, sufficient degrees of freedom result to explain Libet's findings without back-referral. Libet and co-workers did many imaginative and technically demanding experiments and their results (as far as they are supported by further work) should be accommodated by any theory on the relation between physical time and mental (experienced) time. However, we should not be inhibited by his resistance to develop neuroscientifically reasonable theories with a small number of postulated hidden variables, provided the theories are falsifiable and serve as fruitful tools of thought.

The idea of splitting the pathway from cortical electrode to conscious experience in (at least) two serial processes (Gomes, 1998, 2002) is basic to the reasoning of most of Libet's opponents, including Churchland (1981a, 1981b), Glynn (1990, 1991), Dennett and Kinsbourne (1992), and Pocket (2002). Gomes, following the leads of Churchland and Glynn, hypothesizes an intervening integrative process in which the incoming pulses build up a signal until that signal reaches and exceeds a threshold. Then it triggers the next station, which is responsible for the conscious experience and which has its own latency. Pocket (2002) reinterprets the cortical stimulation experiments of Libet and collegues in a similar way. She postulates facilitation of subsequent pulses in a neural process that intervenes between the stimulation electrode and the neurons supporting conscious experiences. Facilitation could also be an additional aspect of the integrative process postulated by Gomes.

Let us translate these ideas in terms of simple but specific neuromimetic models. The discussion centers on the idea that the first neural stage that has to be passed from electrode to NCC is either a kind of stimulus integration process (Gomes, 1998) or a facilitation process (Pocket, 2002) or a bit of both. Integrators, especially of the $\mathrm{RC}$ integrator type (leaky integrators), are quite common in neuroscientific theories. For example, the membrane of a nerve cell is often modeled as a leaky integrator. If the incoming pulse train builds up a signal in such an integrator, this signal might after a while exceed a threshold. The integrator/threshold mechanism could then 


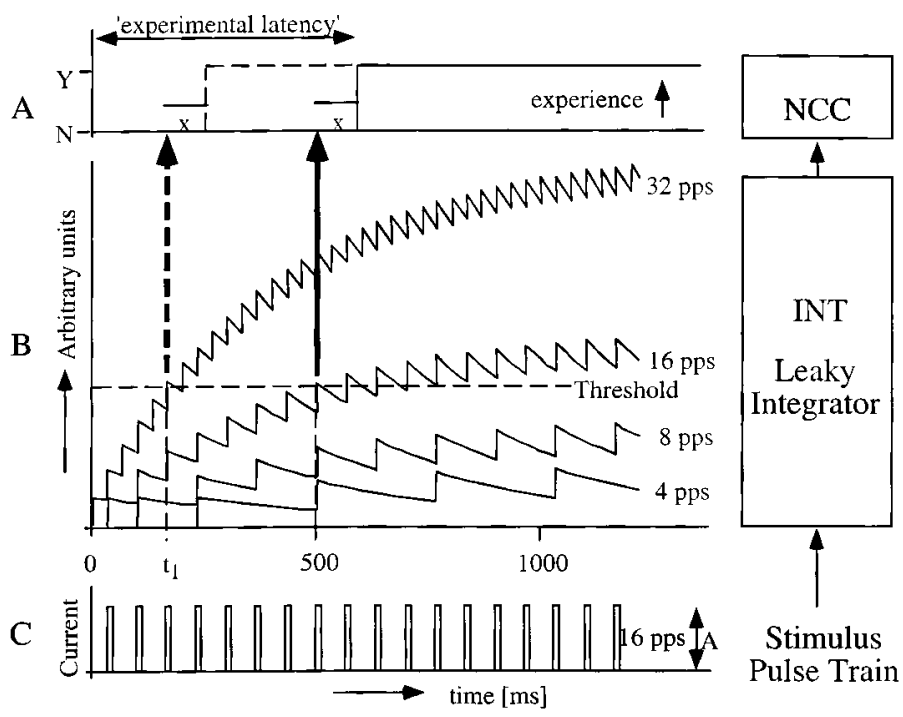

FIG. 1. Part C symbolizes the stimulus pulse train of a typical Libet experiment, while part B shows the response of a leaky integrator to such a stimulus of $16 \mathrm{pps}$ (pulses per second). B also shows integrator responses for other pulse frequencies, which are either too low (4 and $8 \mathrm{pps}$ ) to reach the threshold (horizontal interrupted line) or higher (32 pps) than that of the pulse-train sketched in C. Part A symbolizes the timing of conscious experiences that might result $x$ time units (the "real" latency of Gomes, 1998) after the threshold in B is crossed, for stimulus strengths of both 16 and 32 pps. This type of model explains the shortening of experimental latency for higher stimulus frequencies and eliminates the need for back-referral of conscious experience in time (Gomes, 1998).

generate a message and send it to the NCC. The latency from the generation of this signal to the moment the NCC evokes an experience is called the "real latency" by Gomes (1998).

In Fig. 1 I give my translation of Gomes's ideas in terms of a leaky integrator with threshold detector and Fig. 2A presents a schematic diagram of this system. Fig. 1B clarifies how the frequency of the stimulating pulse train of Fig. 1C influences the experimental (that is overall) latency and why some minimal frequency is required to exceed the threshold. An increase of the pulse amplitudes has the same effect as lowering the threshold (Fig. 1B) and so has a change of gain, $w$, at the input of the integrator. In fact, if the pulse amplitude is $A$ then only the ratio $\Theta=\theta /(w A)$, the normalized threshold, matters. Two parameters, the integration time constant and the normalized threshold, fully characterize this integrator model. Fig. 1A illustrates the timing of experiences for two different stimulation frequencies, where we include the assumption of a "real latency," $x$. The reasoning by Gomes (1998) shows that this general idea is viable and can explain Libet's findings perfectly well without accepting any "back-referral.",

Pocket (2002) proposes facilitation without integration. In Fig. 2B a possible facilitation scheme is given that also uses a leaky integrator ( $\mathrm{RC}$ integrator or rate meter). The pulse amplitudes in Fig. 2B are multiplied by a gain factor $g=c+u$, where $u$ is the output of a leaky integrator fed by the stimulus pulse train. The integrator 


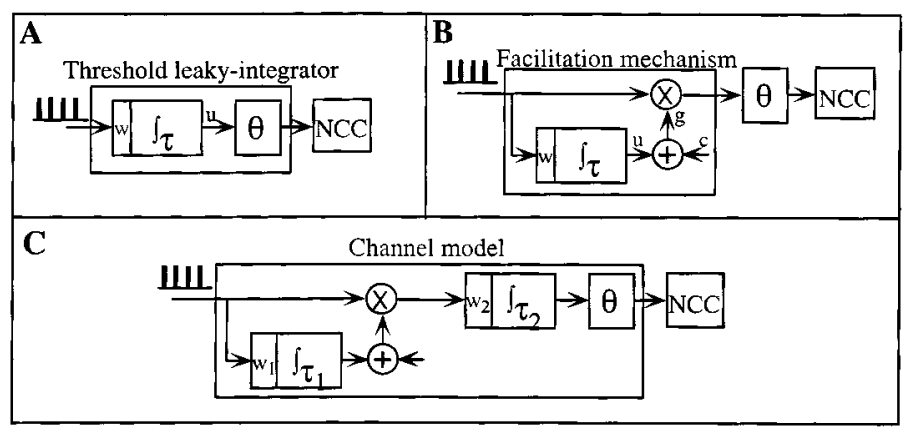

FIG. 2. Part A presents a schematic diagram of the threshold-leaky-integrator system of Fig. 1, whereas part B gives an alternative model (see Pocket, this issue) in which facilitation increases the pulse amplitude of subsequent pulses until the amplitude of the $N$ th pulse of the train exceeds threshold $\theta$ and activates the NCC. Here facilitation is assumed to occur by the increase of a gain factor $c+u$, in which $u$ is the increasing output of a leaky integrator. In part $\mathrm{C}$ the models of A and B are combined. Such a combined model could be hypothesized to transform the delivered external stimuli into a signal that activates the NCC. Each of the channels (neural pathways from external stimulus to internal triggering of the NCC) used in Libet's various experiments could have different parameter values.

signal $u$ grows with increasing pulse frequency or pulse amplitude as in Fig. 1B and consequently the pulse amplitudes at the output of this system will grow with the gain factor $c+u$. It is quite possible of course to place the facilitation box of Fig. 2B in front of the integration box of Fig. 2A to obtain a model as sketched in Fig. $2 \mathrm{C}$. In such a model one can play with the integration time constants $\tau_{1}$ and $\tau_{2}$ to vary the balance between only facilitation, only integration, or both integration and facilitation. If $\tau_{1}$ is very brief there will be no facilitation and if $\tau_{2}$ is brief there will be no integration.

One can characterize a combined facilitation-integration model (Fig. 2C) by three parameters, $\tau_{1}, \tau_{2}$, and $\Theta$, where $\Theta$ is the normalized threshold. Thus we have a general integration-facilitation model that causally connects the stimulus world with the NCC and where the NCC has its own reaction latency. There are three parallel "channels" from the outside world to the NCC, which have been stimulated pairwise in Libet's experiments: A P-channel from the periphery (hand) to the NCC input, a C-channel from a cortical electrode, and an LM-channel from the medial lemniscus to the NCC input. In a final model we also need to include some competition between channels, e.g., mutual inhibition, to explain masking phenomena, especially if the C-channel represents the same body side as the P-channel (Gomes, 1998). Masking models exist in the literature on vision (reviews on visual masking are Fox, 1978, and Bachmann, 1994), but one needs more data than provided by Libet and collegues to design a specific masking model for the system under discussion.

Models as presented in Fig. 2 or by Libet's various opponents are neuroscientifically quite reasonable and do not assume anything out of the ordinary. Gomes (1998) and Pocket (2002) show for their respective models that one can explain Libet's findings without the need for backward referrals of any kind. This means that a mixed model, like the one shown in Fig. 2C, would probably also work. One disadvantage is that these models are underconstrained. Facilitation alone (Pocket, 2002), integra- 
tion alone (Gomes, 1998), or various mixtures are likely to work, possibly for various parameter choices. If facilitation plays a role it could just as well be feedback facilitation rather than the chosen feedforward option of Fig. 2B. Masking needs to be included, but data bearing on the possible implementation are scarce or absent. In short, there are few reasons to prefer one possibility above the other and there are probably many viable alternatives.

That is why, for the time being, Libet is perfectly right if he calls the assumptions proposed by his opponents ad hoc. Yet, the crucial advantage of the theories proposed by Gomes (1998, 2002) and Pocket (2002) is that they eliminate back-referral and can be made precise and empirical. Ironically, there may be no other possible way to falsify Libet's proposal of backward referral of mental time to some early wiggle in the EEG except by showing that one of the competing ad hoc models fits all the neuroscientific facts. This requires more detailed neuroscientific research, but at least we have some models to guide us. Moreover, that work need not be done on humans. One can do similar experiments on awake task-performing monkeys and apply the whole modern-day neuroscientific toolbox to get at the underlying mechanisms. Libet's back-referral theory can only become a serious option if this detailed neuroscientific approach fails to lead to a back-referral-free theory. In Section 3 we see that work on the visual system is already producing neuroscientifically oriented models that circumvent Münchhausenesque operations.

Suppose we find that the conscious experiences studied by Libet lag only $100 \mathrm{~ms}$ behind for normal (rather than liminal) external stimulation and there is no backreferral. The implication would still seem to be that our experiences are ordered according to the neural timing of input signals to the NCC, which track physical time. But, how many independent inputs does the NCC have, and do they all have different latencies? How then are signals with different latencies from all over the body that belong to one event combined into one experience? For example, if you dive into cold water, your visual and auditory inputs soon change abruptly, your fingers hit the water long before your toes get wet, and the skin takes time to get cold. How do we bind all these asynchronous signals into one plunge experience? Are all the signals internally synchronized before reaching the NCC? The reinterpretation of Libet's experiments does not solve Libet's problem, but draws our attention to more interesting aspects of the relation between physical, neural, and mental timing. In vision research some of these aspects have already been studied, so let us take stock.

\section{THE TIMING OF VISUAL PERCEPTS}

\subsection{General Insights}

Many phenomena in visual perception falsify the simple intuition that our experiences relate one-to-one to neural activity in real time. Various forms of time dispersion lead to experiences that have nothing to do with time at all. Let us take a simple but convincing example to illustrate this, the Pulfrich effect. Looking with two eyes at a pendulum moving from left to right, with a weak dark filter in front of one eye, an elliptical path in depth is seen, with the pendulum coming closer during one half of its swing and moving away during the return path. The basic explanation is that 
latencies increase for decreasing luminance, so that the eye with dark filter always sees the pendulum behind the position where the other eye sees it and this spatial parallax (disparity) is translated into depth by our stereovision system (reviewed in Ch. 13 of Howard \& Rogers, 1995). Pulfrich (1922) thus cites knight Gurnemenz in Wagner's Parzival as saying: "Du siehst, mein Sohn, zum Raum wird hier die Zeit" ("You see, my son, here time turns into space"). This is an apt description. The experience of space, or rather depth, is based in this case on a difference in latency of the corresponding signals from the two eyes. The later signal from the eye with the dark filter is not experienced as arriving later at all, so there is no simple oneto-one mapping of neural time on experienced time, at least not for the front-end visual system.

The Pulfrich effect is just one of many examples where sequential neural signals are combined into a nontemporal percept. An everyday experience as motion vision is also based on the combination of neural events that are time-shifted relative to each other. As in the case of the Pulfrich effect I do not attempt to discuss all the possible explanations, but just look at the common core of all proposals. For motion vision this is that the signal from one retinal receptive field $\mathrm{A}$ is first delayed $\tau$ seconds and then combined with the undelayed signal from receptive field $\mathrm{B}$, which is spatially shifted $S$ degrees of visual angle relative to A. Any target that travels the distance $S$ from A to B in $\tau$ seconds will lead to a coincidence in the neuron (motion sensor) that combines their signals multiplicatively, so this neuron is tuned to speed $V=S / \tau$. The idea is that we have whole banks of such motion sensors tuned to a range of speeds and motion directions looking at any region of the visual field. The slower speeds are better represented in the region near the fixation point (the fovea of the retina), the faster speeds are better represented farther out, away from the fovea. The surprising point we need to stress is therefore, again, that the experience of motion is based on the activity of motion sensors that combine signals with a time dispersion. Time differences in the front-end visual system are thus translated into a motion experience, not into an experience-of-time difference. The reverse also happens. Time-to-contact measurements in the visual system probably consist of the combination of size and expansion speed measurements (van de Grind, 1990), so here space and motion turn into time!

The lessons are (1) that latency differences that do not exist in the external world can be generated in the nervous system (Pulfrich effect), but these latency differences do not need to correspond to any timing experience; and (2) that, conversely, latency differences existing at the start of the causal chain in the external world (now at A and later at B) can be combined into one coherent neural signal at some higher processing stage and turned into a primary experience like motion. Gurnemenz might say: "You see, my son, time turns into motion." But in "form-from-motion"' the recoding of temporal dispersion could lead to form perception rather than motion. There is a front-end system, preceding the recoding stages, where the perceptual meaning of a signal is not yet fixed. It could be called the uncommitted part of the nervous system (Koenderink, Kappers, \& van Doorn, 1992). For example, a single receptive field input to a motion sensor in the primary visual cortex does not signify motion. The same receptive field might also be used (read out) by many other visual subsystems, translating its contribution into the experience of form, color, position, 
and so on. The input to V1 and (part of) V1 itself can be viewed as a dynamic database, changing continuously, which is read out by all sorts of specialized modules. These modules recode front-end information and in doing so they each have their own strategies of combining inputs over various module-specific temporal (and also spatial) windows.

Differences in front-end latencies do not simply map onto mental time differences, so they do not represent experiential time differences! Recoding is a hidden operation ("miracle") inside the visual system that wracks our intuitive assumption that neural time and neural time differences map somehow directly onto mental time and mental time differences.

\subsection{Different Latencies for Color, Motion, and Form Perception}

As reviewed by Zeki (1978); Livingstone and Hubel (1984); Felleman and VanEssen (1991); and DeYoe, Felleman, VanEssen, and Mcclendon (1994) the primate visual system is a highly parallel system with spatially dispersed functionally specialized modules for color vision, motion vision, stereovision, form vision, texture vision, object representation, face recognition, and so on. Inevitably such a system must show temporal dispersion in the various specialized modules of responses to one and the same external visual event. The question therefore arises how the perceptual correlate "knows" what to combine with what: the famous "binding problem." If you see a lady in a blue dress and a man in a yellow suit cross the street in opposite directions, how does your brain know that yellow and motion to the left (say) goes with the suit, and blue and motion to the right with the dress, and not vice versa? Since the dress and suit are represented in a brain region different from the color region and motion region one can imagine (1) that the binding occurs first (unconsciously) and the conscious experience after that, perhaps in a common target region (some mention the prefrontal cortex); or (2) that microconsciousness lets you experience the color, motion, and forms in parallel while some other process simultaneously (or somewhat later) "binds" them. In order to solve such riddles, one would like to measure the relative timing of the separate visual experiences and of their possible neuronal correlates. Zeki and collegues, who favor the idea of asynchronous microconsciousnesses (Bartels \& Zeki, 1998; Zeki \& Bartels, 1998a, 1998b, 1999), have looked into these timing problems.

Moutoussis and Zeki (1997) and Zeki and Moutoussis (1997) did psychophysical experiments with two kinds of stimuli side by side on a screen. One half of the screen might show the attribute "motion" in the form of a periodic up-down motion of a checkerboard pattern switching direction every $268 \mathrm{~ms}$. The other half might show a checkerboard of which the light checks changed abruptly from red to green or vice versa every $268 \mathrm{~ms}$ and were always equiluminant to each other and to the gray checks of the motion stimulus. The task of the subject was to decide which attributes were present simultaneously in the two half-fields while the phase of the attribute switching was changed on one side relative to the other. Similarly, orientation changes of lines could be paired with either motion or color changes and horizontal motion with vertical motion. This made it possible to measure the relative timing of perception of these attribute changes. It was found that color change is seen $63 \mathrm{~ms}$ 
before orientation change and orientation change $52 \mathrm{~ms}$ before change of direction. This matched with the finding that color change was seen $118 \mathrm{~ms}$ before the change of direction. The conclusion is (e.g., Moutoussis \& Zeki, 1997, p. 413) that the brain "misbinds"' the different attributes in time and that "it is not equipped with a timecompensator mechanism that could bring the different attributes back into their correct temporal relation.'

One of the reasons why these findings on perceptual timing are remarkable is that the timing of neural signals related to these attributes appears to be entirely different. The fast motion area, V5, receives motion signals with a latency as short as $30 \mathrm{~ms}$ compared with $70 \mathrm{~ms}$ for the color analysis area, V4 (Zeki, 2001). This is the reverse order relative to the perception of color and motion. Neural time and perceptual time do not simply match each other in a one-to-one fashion. The question of how neural time maps onto mental (here perceptual) time is a serious one. It requires empirical study rather than a priori answers. We need to take all of the three separate frames of reference into account, physical time, neural time, and mental time.

\subsection{The Flash-Lag Effect}

When Libet wanted to time the intention to move a finger (see Section 5 and Gomes, 1998, 2002; Pockett, 2002; Trevena \& Miller, 2002) he used a fast rotating clock from which the subjects had to read the decision time and movement time of their finger. This entails reading the position of a moving object at a chosen time, a perceptual feat that proves to be quite tricky. Libet assumes that the observer always sees the moving dial at time $t$ where it is at time $t$ because he adheres to his backreferral hypothesis. Psychophysical studies of the so-called flash-lag effect show that this assumption is quite wrong. In fact the opposite of Libet's hypothesis has been proposed by Nijhawan (1994), who discovered an effect with strong similarity to some almost-forgotten classic visual effects (Fröhlich, 1923, 1929; Hazelhoff, 1924; Metzger, 1932; MacKay, 1958). If a moving object, e.g., a black ring, passes a given position at time $t$, a very brief flash is given, e.g., inside the ring, that coincides in time and space. The flash is then perceived as lagging some distance behind the apparent position of the moving object. Nijhawan $(1994,1997,2001)$ proposes that the nervous system predicts a motion path so that the perceived position of the moving object is close to veridical at all times. Due to its latency it takes a while before the flash is perceived (at its proper position), say at time $t+T$, but in the meantime the moving object is already at a different position, it has moved a distance, $V T$, if $V$ is its speed. Hence the flash-lag effect, which indeed increases linearly with the speed of the moving object (Krekelberg \& Lappe, 1999, 2001). The variable $T$, the lagtime, is on the order of $70-80 \mathrm{~ms}$ in most experiments.

Skilled actions clearly demonstrate predictive mechanisms (motor planning) at work, so prediction mechanisms do exist in the nervous system. However, these are usually assumed to be of a purely motor type. While hitting or catching a ball we can move our arm or body toward an expected future position. Conscious perception is not likely to be involved in the programming and execution of time-critical skilled actions, although it probably is in their initiation and evaluation. So the question is as follows: Does perception use prediction mechanisms? If not, how do we explain 
the flash-lag effect? The findings by Zeki and co-workers, as summarized above, clearly speak against the prediction hypothesis, since motion has the longest perceptual latency of the visual features they compared, more than $100 \mathrm{~ms}$. Unfortunately the Zeki group and the flash-lag students do not appear to read each other's articles. Let us therefore look at opposition against the prediction hypothesis from within the camp of flash-lag research itself.

Whitney and Murakami (1998) and Whitney, Murakami, and Cavanagh (2000) had the object move in one direction until the stationary flash appeared and in the opposite direction from the flash time. The extrapolation hypothesis would predict a mislocalization of the moving object beyond the flash position, but that does not happen. They proposed that the flash percept has a longer latency than the motionposition percept, perhaps because the latter is shortened by recruitment (sensitization) of motion sensors along the motion path (Krekelberg \& Lappe, 1999). This is the latency-difference hypothesis. A hidden assumption appears to be that only the external positions of stimuli causing simultaneous neural signals at some NCC can be compared (see also Section 3.4). One could argue that the position signal of the flash is sustained and that it need not be the start of that signal but could be some later part that is compared to the motion signal. Whitney et al. (2000), however, showed that differences in persistence of the flash and motion signals could not account for the flash-lag effect. They did this by partly masking the flash to reduce its persistence duration. It had no effect. I think one could still argue that some position memory keeps the position signal "online," without remembering the flash appearance itself. Position memory is well known from single-unit studies on the prefrontal cortex (area 46 around the principle sulcus) of task-performing monkeys (Goldman-Rakic, 1995, 1996, 1997; Constantinidis et al., 2001; Wilson et al., 1993). The prefrontal workingmemory region could well be involved in comparing position tags that are not available at the same time.

Brenner and Smeets (2000), like Whitney et al. (2000), studied the flash-lag effect around an abrupt speed change of the moving target. They found that the lag depends on the velocity after, rather than before, the flash. This fits well with the following findings by Eagleman and Sejnowski (2000). Suppose you stop the moving target abruptly at the position and time of the flash. In that case no flash-lag effect occurs, so the preflash path does not seem to play a role in generating it. Now suppose you start the movement at the position and time of the flash. In that case you get a normal flash-lag effect, so it is the postflash trajectory that is responsible for the lag effect. This led to the hypothesis that the flash resets a process of position integration for the moving target. The hypothesis explains why a fresh start of motion after a flash gives the same lag effect as a continuous motion before and after the flash. Further experiments by these authors suggested that the position calculation for a moving stimulus takes about $80 \mathrm{~ms}$ (Eagleman \& Sejnowski, 2000). This reminds us of the Fröhlich effect. Fröhlich $(1923,1929)$ discovered that a slit of light moving at a constant speed from the left edge (say) into a window on a screen is first seen at some distance from the left edge, never at the left edge. It apparently takes some time after the start before the visual system has determined a position signal for a moving target and the calculation involves averaging of a range of previous positions, so that it never gives you the starting position (Krekelberg \& Lappe, 2000). 
The bottom line is that the position tag of a moving stimulus is not something trivial, and indeed why should it be? Scientists who are not aware of the local sign problem (Section 4) might assume that the nervous system only needs to look at the receptor that has just passed the moving target when the flash comes. But the nervous system cannot read the position of a receptor in the retina like an external observer, it needs to "calculate", position labels (Section 4) just as it needs all sorts of operations (calculations) to determine color, motion, and form. Position is a feature like the others (Krekelberg, 2001), not given a priori by the physical position of some activity pattern in the head. Motion also strongly influences the position labeling for other targets even at some distance from the motion (Whitney \& Cavanagh, 2000). The motion aftereffect, an illusory motion percept seen on a static test pattern after prolonged motion adaptation, can displace the apparent position of static targets as well (Snowden, 1998; Nishida \& Johnston, 1999). That spatial position is just as abstract a concept in neural terms as any other feature is also brought home by the findings of Sheth, Nijhawan, and Shimojo (2000), who reported the following. Suppose the color of one disk cycles continuously from, say, red to green and back. A second colored disk is flashed at a certain moment during the cycle, exactly when the first disk has the same color. The color of the flashed disk is then seen to correspond to a previous color in the cycle, so it lags. This is the color version of the flash-lag effect and it works similarly for various other feature changes. Again, it seems fair to conclude that position is a feature, just like color and form.

Eagleman and Sejnowski (2000) coined the hypothesis that the position of a moving stimulus (as determined by the mentioned averaging process) is postdicted, that is, "referred back,' to the time of the flash. Here we have Libet's hypothesis again, albeit for a less sensational time shift. It met with similar opposition (Krekelberg \& Lappe, 2000b; Patel, Ogmen, Bedell, and Sampath, 2000). I also fail to see the reason for such a peculiar assumption. It hinges on the implicit assumption that the perceptual positions of the flash and the moving target can only be compared if their signals arrive exactly simultaneously at some neural "court for position judgement.' (See also 3.4). The calculated position of the moving object is first available $80 \mathrm{~ms}$ after the flash so if all position signals take equal time to reach the court, the motion position is $80 \mathrm{~ms}$ too late to testify. A smart lawyer then antedates this position tag to the arrival time of the flash-position tag. This is what postdiction seems to imply: Position tags are compared only if they have equal time tags. However, if our perceptual "now" is a window of substantial duration, we can be tolerant regarding the 80-ms difference. Within such a broader window of "now" the interpolated position of the moving target and that of the flash differ spatially at the first moment they are both available, and that is all the subject is required to report.

The flash-lag findings can be explained on the basis of latency differences alone, even while sticking to the assumption that position tags are only compared if they have the same time tag (Whitney et al., 2000). A second possibility is to hypothesize that position memory (Goldman-Rakic, 1995, 1996, 1997; Constantinidis et al., 2001; Wilson et al., 1993) plays a role, so that mistuned latencies can be corrected by keeping the early signals online in working memory. In this way one can create a relatively broad time window of consciousness. I like this option best because it enables us to understand how unitary experiences can arise from temporally dispersed 
neural signals, as in the above-mentioned plunge experience. It also eliminates worries about the change of time delays with intensity (Purushothaman, Patel, Bedell, \& Ogmen, 1998). This brief review on the flash-lag phenomenon illustrates how one can relatively easily set up a "playground" for study of the relations between physical time, neural time, and mental time. Most of the reviewed psychophysical experiments can also be done on task-performing monkeys while recording single-unit responses of neurons that are relevant to the tasks and to the hypothesis one wants to test.

The flash-lag and Fröhlich effects are probably a lot more complex than they appear from the above discussion and in about the same way as the effects uncovered by Libet and collegues. Attention might play a role as a latency-shortening process (Kirschfeld \& Kammer, 1999; Baldo \& Klein, 1995; but see Khurana \& Nijhawan, 1995, and Khurana, Watanabe, \& Nijhawan, 2000). Motion deblurring (Burr, 1980) and metacontrast (Kirschfeld \& Kammer, 1999) must also play a role. Moreover, the speed of the target determines which of two motion analysis systems-for fast and for slow motion-dominates (Zeki, 2001; van de Grind, van Hof, van der Smagt, \& Verstraten, 2001; van der Smagt, Verstraten, \& van de Grind, 1999) and we do not know for certain that the reviewed effects hold in the same way for both systems.

\subsection{Temporal Binding and Synchrony}

A peculiar problem arises if we assume that the temporal structure of messages in the nervous system is directly meaningful to the NCC and thus to our percepts. Reasons why this might not be such a great idea are given under Section 4, but here we first look at the consequences. Both in the discussions about Libet's work (Section 2) and when we discussed the flash-lag effect (Section 3.3) it appeared that all parties concerned agree that signals need to reach the $\operatorname{NCC}(s)$ at the same time for their causes to be perceived as simultaneous. Also equal duration of nervous signals was assumed to correspond to equal durations in experience. These assumptions give a special twist to the temporal "binding problem.' It would seem easiest to bind separate signals in various brain areas that belong to one external event (or one percept) if they are somehow synchronized. This viewpoint also appears natural to most students of the EEG because EEG waves are the visible consequences of huge numbers of neurons acting more or less in concert. The fact that there are EEG waves, sometimes even very marked ones, testifies to the existence of forms of synchrony in the nervous system.

The binding problem results from the assertion that we have too many different experiences to accommodate them in a "gnostic-neuron" theory which assumes one cardinal neuron for every experience (Konorski, 1948). Cell assemblies (Hebb, 1949) are a way out if one assumes that assemblies can be formed and broken dynamically so that elements of experience can be combined and recombined with time. The same cells can then contribute to many compound experiences. Von der Malsburg and coworkers (von der Malsburg, 1985, 1995; von der Malsburg \& Schneider, 1986) proposed dynamic coupling on the basis of synchrony - cells that fire together temporarily bind together in an assembly. Electrophysiological support for the idea was reported by Eckhorn et al. $(1988,1993)$, but the idea that synchrony helps solve the 
temporal binding problem was especially made popular by W. Singer and co-workers. A review by Singer followed by an extensive critical evaluation of this temporal binding hypothesis by Shadlen and Movshon, including a complete bibliography, was published recently (Singer, 1999; Shadlen \& Movshon, 1999), so I can be brief about it.

Synchrony can only help in temporal binding if we assume that the NCC preferably or exclusively combines those signals that arrive (almost) simultaneously into one experience. Again this is an assumption that clashes with the findings under Section 3.2 by Zeki and co-workers, but there seems to be no discussion about this. The experimental work by Eckhorn and collegues as well as by Singer and co-workers proves beyond the shadow of a doubt that synchronization occurs, certainly in the early stages of visual processing. Even correlations of firing patterns across large distances were reported. A problem is that the meaning of these phenomena is far from certain, and the binding idea is just one of the possible guesses. Wilder hypotheses exist, for example, the theory by John (2001) or the suggestion of Varela (2000) as cited by John: "the self may be a transient dynamic signature of a distributed array of many brain regions integrated by such synchrony." What is so special about synchronous activity? Synchrony is visible to the external observer, the neuroscientist, but it can only have functional significance if the NCC can also "see" it and interpret it. Without detailed discussion of the inner workings of the NCC (see Section 4) one is forced to conclude that synchrony as "code" implies the assumption of a homunculus with feeling for rhythm.

Kirschfeld (1992) reported oscillations in the gamma frequency band in the insect brain, which should certainly warn us not to read too much in synchrony as far as consciousness or the "self" is concerned. Many processes at the cellular and subcellular level undergo feedback and lateral influences and can easily be made to oscillate. Even spontaneous activity in the retina can harbor oscillations (Przybyszewski et al., 1995). In sensory systems environmental stimuli, including fluorescent light sources in the laboratory (Eysel \& Burandt, 1984), ventilators, ticking clocks, or nearby traffic, can set up oscillatory activity. Blood is pumped through our arteries and one can see the exposed retina and brain pulsate in its rhythm. This might stretch and bend dendrites, thus modulating depolarization (as in pressure phosphenes; Grüsser et al., 1989). Oxygen exchange is rhythmic (breathing) and sets up biochemical waves. The nervous system contains pacemaker cells to drive motoric actions and metabolism. The brain encompasses a bundle of rhythms, but does this have any direct bearing on perceptual binding and consciousness? Can the NCC "see" any of these many rhythms? The debate goes on. In the context of our present debate regarding Libet's problem the lesson must be that the alternatives proposed by Libet's opponents also assume that synchronous arrival of signals at the NCC is of the essence. As explained below I think one should be critical of this assumption.

\section{THE LOCAL SIGN PROBLEM IN SPACE AND TIME}

Lotze (1884) was probably the first to explicitly formulate the problem of "local sign.' In modern formulation (Koenderink, 1984a, 1984b) the local sign problem is that the nervous system cannot see the anatomical layout of its own wiring and thus 
cannot see the spatial order of somatotopic mappings. The orderly arrangement of peripheral receptors and their central projections provide an address book only for the external observer. This obvious address book has misled scientists in believing that the position of a fiber in the brain is also sufficient address information for the brain itself. However, the address tags have to be included in the activity patterns before the brain can process them. Once this is achieved a disordering of the fibers without changing their connectivity would go unnoticed by the owner of the brain. The somatotopic mappings probably enable convenient mapping strategies during embryogenesis and may minimize average lateral interaction distances in the brain. They are also convenient to neuroscientists, but not sufficient to explain the perception of spatial relations. If the spatial address tags are included in the activity patterns and the somatotopic projections are regarded as irrelevant in principle to spatial percepts, the homunculus retires into the wires. The same wiring principles hold in electronic equipment from the tube radio set to the laptop computer. The layout of components and connections is determined by practical considerations of parasitic effects and heat production, but have no bearing on the processing principles.

By solving the local sign problem properly (Koenderink, 1984a, 1984b) one eliminates the "Cartesian theatre" of Dennett's nightmares (Dennett \& Kinsbourne, 1992) without invoking Dennett's "multiple drafts" alternative. Information on the position of retinal receptive fields is inherent in the overlap and inclusion relations and thus in the correlation structure of cortical activity patterns. From this correlation structure the construction of an explicit internal (neural) geometry that relates systematically to the geometry of external objects and spaces is possible in principle (Koenderink, 1990, 1993). It is not known in detail how the nervous system solves this problem, nor what kinds of internal geometries result, since few neuroscientists have recognized the problem and worked on it.

Local sign problems also arise when we think of the neural geometry of time. In the same way that the existence of an orderly array of retinal receptors does not solve the (spatial) local sign problem, the presence of an inborn internal clock in organisms does not explain experiential time. The biological clock generates a control signal for bodily homeostasis, but this homeostasis cannot be allowed to be independent of outside events. It would be rather inconvenient to fall asleep while running away from a predator. Thus, the nervous system needs to contain or construct the continuum of mental time from the multidimensional stream of neural events caused by outside events. Methods to do this have also been presented by Koenderink $(1988,2002)$. The point here is that to construct the mental time continuum we need events of various durations and starting and end times (speaking in terms of the external clock). Working memory keeps many past events "online" (Goldman Rakic, 1995, 1996); new events stream in and the present, or "Now," is contained in the successive order relations of these neural events. In this view there is no need at all for synchrony.

All organisms show circadian rhythms and usually the Sun is the ultimate pacemaker ("Zeitgeber') for these rhythms. The biological clock responsible for this rhythm has been studied extensively from the biochemical to the neuronal level (e.g., in the suprachiasmatic nucleus). Every textbook on biology or physiology presents the details. Here I only want to draw the conclusion from all this that some form of "physical" clock is present in the brain. This is not controversial. The question is 
whether this clock is read and interpreted by brain regions involved in perception and, if so, how. Suppose, by way of a thought experiment, that clock ticks at regular intervals in the millisecond range would be available everywhere in the brain. This would make it possible to count clock ticks, for example, with resettable leaky integrators (like in Fig. 1, but without the threshold) of which the output is a function of the number of clock ticks since the most recent reset. Then duration signals could be made available in any brain region by resetting the counter at "feature-X-on" and sending its output to the NCC at "feature-X-off." These count numbers, perhaps translated into a rate code, would be "symbolic" duration tags, since they express physical duration in something else than neural signal duration. These tags could enter the NCC together with the visual feature information $\mathrm{X}$ so that we perceive them together as object/event $\mathrm{X}$ of duration $T$. Interestingly, we could get the binding problem again, but now for duration tag and event information. A way out is to multiplex duration tag and event information into one neural signal (code) that enters the NCC. Smart coding could solve this part of the binding problem without burdening the NCC.

The alternative to having a smart coding front end (signaling temporal and spatial information in symbolic form) is to have a smart NCC and dumb front end. Suppose that the NCC itself contains a map of the input wires (to solve the local sign problem and measure position $S$ ), duration counters (to measure $T$ ), and a clock (to measure signal arrival time $t$ ). By the way, a bank of duration counters could serve as a clock, integrators with lower input weights for the longer duration counts, and so on. In this way the NCC could even "know" the timing of external triggers like the Sun or your breakfast. How then can the NCC "bind" sub-experiences $X_{1}$ and $X_{2}$ ? Four basic pieces of information are required and they now have to be determined by the NCC: place of occurrence $(S)$, physical time of occurrence $(t)$, physical duration $(T)$, and event-type $(X)$. It is clear that the NCC clock is of no help at all! The NCC has no way to know the physical times of occurrence of the two subevents; its clock only indicates the arrival time at the NCC, not the stimulation time of the receptors. Thus the NCC would at least need to know the whole route of the subevent signals and the concomitant delays to bind the incoming signals properly. This is what Libet postulates. In our terms: The NCC reads an early wiggle in the EEG to get at the physical time of occurrence of input events so that it can back-refer the signal arrival times to physical times.

How do such sticky conceptual problems arise? My diagnosis is simple: If you suppose consciousness is very smart and solves all problems of time and space labeling/binding, you paint yourself into this corner. It is a requirement of a good theory to postulate smart recoding as early as possible on the neural pathways from receptors to NCC. These smart recoding stages should construct symbolic space and time tags that are either multiplexed or sent along equal-delay pathways to the NCC.

A smart NCC, remote from the receptor layers of the body and senses, needs to be almost clairvoyant to solve the local sign and temporal binding problems. A "dumb" NCC that does not itself solve these problems necessitates a smart intervening process between the external world and the NCC. I think this dichotomy is at the heart of many discussions on consciousness. For Cartesians, mystics, and people tending to give consciousness an almost supernatural status, it is "natural" to make 
the NCC extremely smart and the rest of the nervous system rather dumb. In their camp we find reliance on the assumption that space and time parameters of nervous activity are just as meaningful to the NCC as they are to the external observer. Others, mainly neuroscientists seeing that they cannot place an electrode in the NCC yet, prefer to assume that the more accessible parts of the nervous system are smart, leaving a less impressive role for the NCC. They should probably start looking for symbolic space and time codes. So far few empirical scientists have sketched out a complete hypothesis of smart recoding, so almost all of this work still has to be done.

It is interesting in this connection how the assumption of synchronicity appeals to scientists who like the smart-NCC hypothesis. They find themselves in the company of mystics, who have always known that consciousness stands for "smart" and neuronal networks for "dumb." John and Varela, as cited above, are apparently in this camp, and I think Libet is as well. The discoverer of the EEG, Hans Berger, also placed himself squarely in this camp (Beyerstein, 1999). The opponents have argued against the symptoms of the smart-NCC assumption (e.g., Dennett \& Kinsbourne, 1992), but it is time to show with empirical neuroscience methods how smart the intervening processes really are. This will distribute the smartness more evenly and leave to the NCC some relatively simple task like coordination of the actions of other parts of the nervous system.

\section{THE POSSIBLE CAUSAL ROLE OF CONSCIOUSNESS AND "FREE" WILL}

I think most neuroscientists realize that "free will" is a misnomer. There is hardly anything "free" about it. Whatever you decide, it comes out of the brain using information that is there, sometimes combined with immediate stimulation from the environment. This does not mean, however, that there is no choice in the preparation of decisions, just that the choice is limited and partly based on fixed decision criteria that we inherited and learned. It is further conceivable that if this decision process cannot reach a conclusion, it "throws dice." Neuronal networks or even single neurons can easily generate noise, so a random-choice generator does not seem to be beyond the capabilities of our brain. In the present discussion we limit ourselves to simple voluntary acts like moving a finger or a hand. Libet, Gleason, Wright, and Pearl (1983) asked subjects to make a spontaneous voluntary hand movement at a random time and measured the corresponding "Readiness Potential", (RP), which predicts the action. The decision times were measured in a kind of flash-lag experimental setup: The subject reported the position of a fast-moving dial at the moment of the decision.

We have just seen under Section 3.3 that a moving dial will appear ahead of the position it had at the moment the report is requested (the analog of flash time presented under Section 3.3). Thus the decision-time reports of the subjects will appear to be $70-80 \mathrm{~ms}$ closer in time to their hand movement than they in fact are. This lag time was not taken into account. Libet et al. $(1982,1983)$ found that the average reported time of the decision to move was $200 \mathrm{~ms}$ before the recorded movement. The average onset time of the RP was $500 \mathrm{~ms}$ before the movement, so the RP leads the feeling that one takes a decision by $300-350 \mathrm{~ms}$. Due to the flash-lag effect we 
need to subtract $70-80 \mathrm{~ms}$ from this value, so let us conclude that the RP starts about $250 \mathrm{~ms}$ before the subject has the feeling that he/she decides to move. In itself it is certainly conceivable that many or most of our decisions, which are presumably based on internal nonconscious information, momentary sensory data, and unconscious choice algorithms, are taken before we become aware of them. The question is whether Libet's results prove this to be the case.

Trevena and Miller (2002) argue that the averaging of RPs leads to an apparent shift to earlier times: the smearing artifact. For example, if you average $N$ waves with onset times varying from 20 to $200 \mathrm{~ms}$, the average will start around $20 \mathrm{~ms}$, even if such short latencies are a very small minority. This is a mathematical truth, so one should actually look at the temporal order of RP and voluntary decision from trial to trial. Trevena and Miller did an independent experiment to check the influence of this potential artifact, but found that it could not explain the decision lag (relative to the RP). The RP onset not only precedes the mean decision time but also the earliest decision times. This supports the conclusion of Libet et al. (1982, 1983): RPs precede the reported times of the decisions to act. Such a finding leaves us with two options. Either we accept the conclusion of Libet and collegues that the decision is taken unconsciously or we argue that the RP does not reflect a decision to move at all. The idea that unconscious decisions precede their corresponding conscious decision sounds more interesting, of course, and it would be gratifying to behaviorists. But, before throwing our intuitions about conscious decisions in the wastebasket, we are obliged to exclude the "normal-science" hypothesis that conscious decisions can (sometimes?) be causes of our actions. This step was not undertaken by Libet et al. (1982, 1983), but Trevena and Miller conducted an important additional test.

They argue that the RP reflects not only the decision to move, but also other preparatory activities. Perhaps its initial part reflects the preparation to decide rather than the preparation to move. Trevena and Miller therefore repeated the basic experiment but now using the lateralized readiness potential (LRP), which, as they argue, reflects the decision process itself. It indicates whether the subject is going to move the right or the left hand, so the instruction was to choose randomly between a left or right hand motion at random moments in time. Now it was found that some $20 \%$ of the decisions to move preceded the onset of the LRPs. Since Trevena and Miller also used clock readings by the subjects to time the voluntary decisions, we should shift the decisions to earlier times (flash-lag effect). This means that a much larger proportion than $20 \%$, perhaps almost $100 \%$, must have preceded their LRP. Add to this the smearing artifact, which might make the LRP appear earlier than it did, and the conclusion can be that the decisions might be the "causes" of the movements, since they preceded the movement preparation potentials.

This is a nice story and it might well be right. Yet, it is hard to feel absolutely certain about the conclusions because they hinge strongly on the interpretation of EEG waves, which is not unproblematic. But, I think there is also a theoretical reason why we should not accept the interpretation of Libet et al. (1982, 1983; Libet, 1985) at face value. The subjects agree to play a game in which they need to do something at random moments in time. Why not just assume that this decision starts some nonconscious random generator, after which the subjects check with every randomly generated action signal to see if it is still relevant? Surely we do not want a "will" 
that has to decide on every nitty-gritty detail of neural processing. We make conscious decisions about main events, not details. While playing tennis you might decide to try to smash the ball, but when the opportunity arises the smash just occurs-you do not think about it at all. You realize only afterward that this was the outcome of your conscious decision of perhaps 5 min ago. Whether Libet's idea of nonconscious decisions that are only monitored after the fact bothers you depends on your view of conscious processes in general. Suppose consciousness is only monitoring and controlling the main lines of ongoing processes, is never in a hurry, and only gives global directions. Then the particular experiments of Libet et al. $(1982,1983)$ and Trevena and Miller (2002) do not measure the voluntary initiation of actions, but the conscious monitoring of an ongoing process that was started voluntarily when the subject sat down to join the game.

Again we see that the interpretation hinges on your view of the NCC. Is it smart and always alert, following every detail of your perceptions and actions, or rather is it dumb and lazy, only just capable of giving a hint here and there and picking up curious unexpected tickles or sounds? Opting for the latter means that even the results of Libet et al. (1982, 1983), if taken at face value, do not support anything like the conclusions they formulated. The subjects may have decided to just monitor the nonconsciously generated commands to move given by a neural network that was earlier consciously commanded to generate these events at random. I like this kind of model and have formulated it elsewhere more extensively (van de Grind, 2001). The idea is that conscious experiences are the result of actions of our Brain Operating System (BOS). The BOS is necessary since a complex parallel system like the brain requires coordination. While meeting a lion it would be disastrous if one module would decide to run, another to hide, and yet another to try to remember a nephew's name. The BOS handles priority problems, smoothes conflicting decisions, gives interrupts or resets, and handles other messages between modules and from the modules to the external world or vice versa, but only insofar as these processes cannot run automatically for some reason or other. The BOS is just as smart or dumb as any other functional module of your brain, not Descartes's omniscient homunculus.

\section{REFERENCES}

Bachmann, T. (1994). Psychophysiology of visual masking. Commack, NY: Nova Science.

Baldo, M. V., \& Klein, S. A. (1995). Extrapolation or attention shift? Nature, 378, 565-566.

Bartels, A., \& Zeki, S. (1998). The theory of multistage integration in the visual brain. Proceedings of the Royal Society of London B, 265, 2327-2332.

Beyerstein, B. L. (1999). Pseudoscience and the brain: Tuners and tonics for aspiring superhumans. In S. Della Salla (Ed.), Mind Myths, pp. 59-82. Chichester: Wiley.

Brenner, E., \& Smeets, J. B. J. (2000). Motion extrapolation is not responsible for the flash-lag effect. Vision Research, 40, 1645-1648.

Burr, D. (1980). Motion smear. Nature, 284, 164-165.

Churchland, P. S. (1981a). On the alleged backwards referral of experience and its relevance to the mind-body problem. Philosophy of Science, 48, 165-181.

Churchland, P. S. (1981b). Discussion: The timing of sensations: Reply to Libet. Philosophy of Science, 48, 492-497. 
Constantinidis, C., Franowicz, M. N., \& Goldman-Rakic, P. S. (2001). The sensory nature of mnemonic representation in the primate prefrontal cortex. Nature Neuroscience, 4, 311-316.

Dennett, D. C., \& Kinsbourne, M. (1992). Time and the observer: The where and when of consciousness in the brain. Behavioral and Brain Sciences, 15, 183-247.

DeYoe, E. A., Felleman, D. J., Vanessen, D. C., \& Mcclendon, E. (1994). Multiple processing streams in occipitotemporal visual cortex. Nature, 371, 151-154.

Eagleman, D. M., \& Sejnowski, T. J. (2000). Motion integration and prediction in visual awareness. Science, 287, 2036-2038.

Eckhorn, R., Bauer, R., Jordan, W., Brosch, M., Kruse, W., Munk, M., \& Reitboeck, H. J. (1988). Coherent oscillations: A mechanism for feature linking in the visual cortex? Biological Cybernetics, 60, 121-130.

Eckhorn, R., Frien, A., Bauer, R., Woelbern, T., \& Kehr, H. (1993). High frequency 60-90 Hz oscillations in primary visual cortex of awake monkey. NeuroReport, 4, 243-246.

Eysel, U. T., \& Burandt, U. (1984). Fluorescent tube light evokes flicker responses in visual neurons. Vision Research, 24(9), 943-948.

Felleman, D. J., \& VanEssen, D. C. (1991). Distributed hierarchical processing in primate cerebral cortex. Cerebral Cortex, 1, 1-47.

Fox, R. (1978). Visual masking. In R. Held, W. W. Leibowitz, \& H.-L. Teuber (Eds.), Handbook of sensory physiology, Vol. VIII, pp. 629-653. Berlin: Springer-Verlag.

Fröhlich, F. W. (1923). Über die Messung der Empfindungszeit. Zeitschrift für Sinnesphysiologie, 54, $58-78$.

Fröhlich, F. W. (1929). Die Empfindungszeit. Jena: Verlag Gustav Fischer.

Glynn, I. M. (1990). Consciousness and time. Nature, 348, 477-479.

Glynn, I. M. (1991). Glynn replies. Nature, 352, 27.

Goldman-Rakic, P. S. (1995). Cellular basis of working memory. Neuron, 14, 477-485.

Goldman-Rakic, P. S. (1996). The prefrontal landscape: Implications of functional architecture for understanding human mentation and the central executive. Philosophical Transactions of the Royal Society of London B, 351, 1445-1453.

Goldman-Rakic, P. S. (1997). Space and time in the mental universe. Nature, 386(6625), 559-560.

Gomes, G. (1998). The timing of conscious experience: A critical review and reinterpretation of Libet's research. Consciousness and Cognition, 7, 559-595.

Gomes, G. (2002). Problems in the timing of conscious experience. Consciousness and Cognition, 11, 191-197.

van de Grind, W. A. (1990). Smart mechanisms for the visual evaluation and control of self-motion. In R. Warren \& A. H. Wertheim (Eds.), Perception and control of self motion, pp. 357-398. Hillsdale, NJ: Erlbaum.

van de Grind, W. A. (2000). The neuronal basis of conscious experiences in the animal kingdom. Vlaams Diergeneeskundig Tijdschrift, 69, 392-400.

van de Grind, W. A., van Hof, P., van der Smagt, M. J., \& Verstraten, F. A. J. (2001). Slow and fast visual motion channels have independent binocular rivalry stages. Proceedings of the Royal Society of London B, 268, 437-443.

Grüsser, O.-J., Grüsser-Cornehls, U., Hagner, M., \& Przybyszewski, A. (1989). Purkinje's description of pressure phosphenes and modern neurophysiological studies on the generation of phosphenes by eyeball deformation. Physiologia Bohemoslovaca, 38, 289-309.

Hazelhoff, F. F. (1924). Die Wahrnehmungszeit (I). Zeitschrift für die Psychologie und Physiologie der Sinnesorgane, 97, 174-190.

Hebb, D. O. (1949). The organization of behavior. New York: Wiley.

Howard, I. P., \& Rogers, B. J. (1995). Binocular vision and stereopsis. New York/Oxford: Oxford Univ. Press/Clarendon Press.

John, E. R. (2001). A field theory of consciousness. Consciousness and Cognition, 10, 184-213. 
Khurana, B., \& Nijhawan, R. (1995). Extrapolation or attention shift?: Reply. Nature, 378, 566.

Khurana, B., Watanabe, K., \& Nijhawan, R. (2000). The role of attention in motion extrapolation: Are moving objects 'corrected' or flashed objects attentionally delayed? Perception, 29, 675-692.

Kirschfeld, K. (1992). Oscillations in the insect brain: Do they correspond to the cortical gamma-waves of vertebrates? Proceedings of the National Academy of Sciences of the USA, 89, 4764-4768.

Kirschfeld, K., \& Kammer, T. (1999). The Fröhlich effect: A consequence of the interaction of visual focal attention and metacontrast. Vision Research, 39, 3702-3709.

Koenderink, J. J. (1984a). The concept of local sign. In A. J. van Doorn, W. A. van de Grind, \& J. J. Koenderink (Eds.), Limits in perception, pp. 495-547. Utrecht, The Netherlands: VNU Science Press.

Koenderink, J. J. (1984b). Simultaneous order in nervous nets from a functional standpoint. Biological Cybernetics, 50, 35-41.

Koenderink, J. J. (1988). Scale time. Biological Cybernetics, 58, 159-162.

Koenderink, J. J. (1990). The brain as a geometry machine. Psychology Research, 52, 122-127.

Koenderink, J. J. (1993). Embodiments of geometry. In A. Aertsen (Ed.), Brain theory, pp. 3-28. Amsterdam: Elsevier.

Koenderink, J. J. (2002). Continua in vision. In L. Albertazzi (Ed.), Unfolding perceptual continua, Amsterdam: J. Benjamins.

Koenderink, J. J., Kappers, A., \& van Doorn, A. J. (1992). Local operations: The embodiment of geometry. In G. A. Orban \& H.-H. Nagel (Eds.), Artificial and biological vision systems. Brussels: ESPRIT Basic Research Series.

Konorski, J. (1948). Conditioned reflexes and neuron organization. London: Cambridge Univ. Press.

Krekelberg, B. (2001). The persistence of position. Vision Research, 41, 529-539.

Krekelberg, B., \& Lappe, M. (1999). Temporal recruitment along the trajectory of moving objects and the perception of position. Vision Research, 39, 2669-2679.

Krekelberg, B., \& Lappe, M. (2000). A model of the perceived relative positions of moving objects based upon a slow averaging process. Vision Research, 40, 201-215.

Krekelberg, B., \& Lappe, M. (2000b). The position of moving objects. Science, 289, 1107.

Krekelberg, B., \& Lappe, M. (2001). Neuronal latencies and the position of moving objects. Trends in Neuroscience, 24, 335-339.

Libet, B. (1985). Unconscious cerebral initiative and the role of conscious will in voluntary action. The Behavioral and Brain Sciences, 8, 529-566.

Libet, B. (2000). Time factors in conscious processes: Reply to Gilberto Gomes. Consciousness and Cognition, 9, 1-12.

Libet, B., Wright, E. W., \& Gleason, C. A. (1982). Readiness-Potentials preceding unrestricted 'spontaneous' vs pre-planned voluntary acts. Electroencephalography and Clinical Neurophysiology, 54, $322-335$.

Libet, B., Gleason, C. A., Wright, E. W., \& Pearl, D. K. (1983). Time of conscious intention to act in relation to onset of cerebral activity (Readiness-Potential): The unconscious initiation of a freely voluntary act. Brain, 106, 623-642.

Livingstone, M. S., \& Hubel, D. H. (1988). Segregation of form color movement and depth: Anatomy physiology and perception. Science, 240, 740-749.

Lotze, H. (1884). Mikrokosmos. Leipzig: Hirzel Verlag.

von der Malsburg, C. (1985). Nervous structures with dynamical links. Berichte der BunsenGes ellschaft-Physical Chemistry, 89, 703-710.

von der Malsburg, C. (1995). Binding in models of perception and brain function. Current Opinion in Neurobiology, 5, 520-526.

von der Malsburg, C., \& Schneider, W. (1986). A neural cocktail-party processor. Biological Cybernetics, 54, 29-40. 
MacKay, D. M. (1958). Perceptual stability of a stroboscopically lit visual field containing self-luminous objects. Nature, 181, 507-508.

Metzger, W. (1932). Versuch einer gemeinsamen Theorie der Phänomene Fröhlichs und Hazelhoffs und Kritik ihrer Verfahren zur Messung der Empfindungszeit. Psychologische Forschung, 16, 176-200.

Moutoussis, K., \& Zeki, S. (1997). Functional segregation and temporal hierarchy of the visual perceptive systems. Proceedings of the Royal Society of London B, 264, 1407-1414.

Nijhawan, R. (1994). Motion extrapolation in catching. Nature, 370, 256-257.

Nijhawan, R. (1997). Visual decomposition of colour through motion extrapolation. Nature, 386, 6669.

Nijhawan, R. (2001). The flash-lag phenomenon: Object and eye movements. Perception, 30, 263-282.

Nishida, S., \& Johnston, A. (1999). Influence of motion signals on the perceived position of spatial patterns. Nature, 397, 610-612.

Patel, S. S., Ogmen, H., Bedell, H. E., \& Sampath, V. (2000). Flash-lag effect: Differential latency, not postdiction. Science, 290, 1051.

Pocket, S. (2002). On subjective back-referral and how long it takes to become conscious of a stimulus: A reinterpretation of Libet's data. Consciousness and Cognition, 11, 144-161.

Pöppel, E. (1978). Time perception. In R. Held, W. W. Leibowitz, H.-L. Teuber (Eds.), Handbook of sensory physiology, Vol. VIII, pp. 713-729. Berlin: Springer-Verlag.

Przybyszewski, A. W., Lankheet, M. J. M., \& van de Grind, W. A. (1995). Irregularities in spike trains of cat retinal ganglion cells. In W. Ditto, L. Pecora, M. Shlesinger, M. Spano, \& S. Vohra (Eds.), Proceedings of the 2nd experimental chaos conference, pp. 218-225. Singapore: World Scientific.

Pulfrich, C. (1922). Die Stereoskopie im Dienste der isochromen und heterochromen Photometrie, Die Naturwissenschaften 25, 553-564; 26, 569-574; 27, 596-601; 33, 714-722; 34, 735-743; 35, 751761.

Purushothaman, G., Patel, S. S., Bedell, E., \& Ogmen, H. (1998). Moving ahead through differential visual latency. Nature, 396, 424.

Shadlen, M. N., \& Movshon, J. A. (1999). Synchrony unbound: A critical evaluation of the temporal binding hypothesis. Neuron, 24, 67-77.

Sheth, B. R., Nijhawan, R., \& Shimojo, S. (2000). Changing objects lead briefly flashed ones. Nature Neuroscience, 3, 489-495.

Singer, W. (1999). Neuronal synchrony: A versatile code for the definition of relations? Neuron, 24, 49-65.

van der Smagt, M. J., Verstraten, F. A. J., \& van de Grind, W. A. (1999). New transparent motion aftereffect. Nature Neuroscience, 2, 595-596.

Snowden, R. J. (1998). Shifts in perceived position following adaptation to visual motion. Current Biology, 8, 1343-1345.

Trevena, J. A., \& Miller, J. (2002). Cortical movement preparation before and after a conscious decision to move. Consciousness and Cognition, 11, 162-190.

Varela, F. J. (2000). Neural synchrony and consciousness: Are we going somewhere? Consciousness and Cognition, 9(22), S26-S27.

Whitney, D., \& Cavanagh, P. (2000). Motion distorts visual space: Shifting the perceived position of remote stationary objects. Nature Neuroscience, 3, 954-959.

Whitney, D., \& Murakami, I. (1988). Latency difference, not spatial extrapolation. Nature Neuroscience, 1, 656-657.

Whitney, D., Murakami, I., \& Cavanagh, P. (2000). Illusory spatial offset of a flash relative to a moving stimulus is caused by differential latencies for moving and flashed stimuli. Vision Research, 40, $137-149$.

Wilson, F. A. W., Scalaidhe, S. P. O., \& Goldman-Rakic, P. S. (1993). Dissociation of object and spatial processing domains in primate prefrontal cortex. Science, 260, 5116, 1955-1958.

Zeki, S. (1978). Functional specialisation in the visual cortex of the monkey. Nature, 274, 423-428. 
Zeki, S. (2001). Localization and globalization in conscious vision. Annual Review of Neuroscience, 24, 57-86.

Zeki, S., \& Bartels, A. (1998a). The asynchrony of consciousness. Proceedings of the Royal Society of London B, 265, 1583-1585.

Zeki, S., \& Bartels, A. (1998b). The autonomy of the visual systems and the modularity of conscious vision. Philosophical Transactions of the Royal Society of London B, 353, 1911-1914.

Zeki, S., \& Bartels, A. (1999). Toward a theory of visual consciousness. Consciousness and Cognition, 8, 225-259.

Zeki, S., \& Moutoussis, K. (1997). Temporal hierarchy of the visual perceptive systems in the Mondrian world. Proceedings of the Royal Society of London B, 264, 1415-1419. 